ISSN 1936-5098

CAE Working Paper \#07-05

On Competitive Equitable Paths under Exhaustible Resource Constraints:

The Case of a Growing Population

by

Tapan Mitra

April 2007 


\title{
On Competitive Equitable Paths under Exhaustible Resource Constraints: The Case of a Growing Population ${ }^{1}$
}

\author{
Tapan Mitra²
}

\begin{abstract}
The paper examines the nature of competitive paths in an exhaustible resource model, which allows for growing population. For competitive paths which are equitable in the sense that the per capita consumption level is constant over time, the implicit investment rule is derived. This is seen to be a generalization of Hartwick's rule, obtained in the case of a stationary population. It is also shown that the existence of a competitive equitable path implies that population can experience at most quasi-arithmetic growth.

Journal of Economic Literature Classification Numbers: D90, O11, O41, Q32.

Key words: exhaustible resource, intertemporal equity, Hotelling's rule, Hartwick's rule, quasi-arithmetic population growth.

\footnotetext{
${ }^{1}$ This paper is dedicated to the memory of Koji Shimomura, whose untimely death is a major loss to the economics profession. He was a wonderful person, gentle and unassuming, and I have always felt it a great privilege to have made his acquaintance. I would like to thank Geir Asheim, Wolfgang Buchholz, John Hartwick and Cees Withagen for their comments on an earlier version of this paper.
}

${ }^{2}$ Department of Economics, Cornell University, Ithaca, NY 14853, USA; E-mail: tm19@cornell.edu
\end{abstract}




\section{Introduction}

The purpose of this paper is to examine the nature of competitive equitable paths, when exhaustible resources are essential factors of production, allowing for population growth.

The literature has almost exclusively focused on the case of zero population growth. However, there has been considerable interest recently, from the view point of sustainable development, in examining whether population growth is consistent with the objective of attaining equitable paths (with constant positive per-capita consumption). ${ }^{3}$

In an early precursor to this literature, Mitra (1983) had examined this issue in a discretetime exhaustible resource model. The current paper can be considered to be a continuoustime counterpart of the earlier one, which can be used to provide sharper results on two aspects of the framework with population growth, which have come to the forefront of recent discussion.

The first aspect involves the precise nature of the investment rule implicit in competitive equitable paths. In the absence of population growth, it is known that if a path is competitive and equitable, then the value of net investment, in both the produced capital good, and the non-produced capital good (the exhaustible resource), must equal zero at each date (see Buchholz, Dasgupta and Mitra (2005)). This investment rule is known as Hartwick's rule. However, in the presence of exogenous population growth (that is not restricted in any ad hoc manner) the appropriate investment rule implied by competitive equity is not known. In Theorem 1 of Section 4, we derive this invetment rule, which is seen to be an appropriate generalization of Hartwick's rule. ${ }^{4}$

The second aspect involves the parametric restrictions necessary for the existence of a competitive equitable path. In the framework of our model, but without population growth, Solow (1974) obtained the parametric restriction for competitive equity that the capital coefficient must exceed the resource coefficient in the (assumed Cobb-Douglas) production function. In the current framework, which allows for growing (as well as stationary) population, the additional parameters are precisely the exogenously specified population path. In Theorem 2 of Section 5, we show that the existence of a competitive equitable path implies that population be restricted to at most quasi-arithmetic growth. ${ }^{5}$

\section{Preliminaries}

\subsection{An Exhaustible Resource Model}

This is a model with one produced good, which serves as both the capital as well as the consumption good. This good is produced with capital $(K)$, an exhaustible resource $(R)$, and labor $(N)$. The production function $F$ is of the Cobb-Douglas form:

$$
F(K, R, N)=K^{\alpha} R^{\beta} N^{(1-\alpha-\beta)} \text { for }(K, R, N) \geq 0
$$

\footnotetext{
${ }^{3}$ See Asheim, Buchholz, Hartwick, Mitra and Withagen (2006) for some recent results, as well as references to some of the literature that has developed.

${ }^{4}$ Section 4 contains a more thorough discussion relating our theorem to the earlier literature, and consequently our exposition here is deliberately brief.

${ }^{5} \mathrm{~A}$ discussion of some of the background literature, dealing with the interest in quasi-arithmetic (rather than geometric) population growth in the context of the exhaustible resource model, can be found in Section 5.
} 
where $\alpha>0, \beta>0$ and $(\alpha+\beta)<1$.

Labor is taken to be equal to the population $(N)$, and is assumed to be exogenously given, and non-decreasing over time:

$$
\dot{N}(t)=g(t) N(t) \text { for } t \geq 0 ; N(0)>0
$$

where $g(t) \geq 0$ for $t \geq 0$ represents the growth rate of population, and is assumed to be a continuous function of $t$. We normalize $N(0)$ to 1 , and we also assume that there is some $g>0$, such that $g(t) \leq g$ for all $t \geq 0$.

\subsection{Equitable and Competitive Paths}

A path from initial stock $(K, S)$ in $\mathbb{R}_{+}^{2}$ is described by the functions $(K(t), R(t), C(t))$, where $K(\cdot):[0, \infty) \rightarrow \mathbb{R}_{+}, R(\cdot):[0, \infty) \rightarrow \mathbb{R}_{+}$, and $C(\cdot):[0, \infty) \rightarrow \mathbb{R}_{+}$, such that $K(t), R(t), C(t)$ are continuously differentiable functions of $t$, and satisfy:

$$
\left.\begin{array}{l}
\text { (a) } C(t)=F(K(t), R(t), N(t))-\dot{K}(t) \text { for } t \geq 0 \\
\text { (b) } \int_{0}^{\infty} R(t) d t \leq S \text { and } \\
\text { (c) } K(0)=K
\end{array}\right\}
$$

We associate with a path $(K(t), R(t), C(t))$ from $(K, S)$ in $\mathbb{R}_{+}^{2}$ a function $(E(t))$ given by:

$$
E(t)=\int_{t}^{\infty} R(s) d s
$$

A path $(K(t), R(t), C(t))$ from $(K, S)$ in $\mathbb{R}_{+}^{2}$ is called interior if $K(t)>0, R(t)>0$ and $C(t)>$ 0 for $t \geq 0 .{ }^{6}$

A path $(K(t), R(t), C(t))$ from $(K, S)$ is called equitable if $(C(t) / N(t))$ is constant over time. It is called inefficient if there is another path $\left(K^{\prime}(t), R^{\prime}(t), C^{\prime}(t)\right)$ from $(K, S)$, such that $C^{\prime}(t) \geq C(t)$ for $t \geq 0$, and:

$$
C^{\prime}(\tau)>C(\tau) \text { for some } \tau \geq 0
$$

Note that since $C(t)$ is continuous in $t$, the requirement (4) in fact implies that there is an interval $[\tau, \tau+\varepsilon]$, with $\varepsilon>0$, such that:

$$
C^{\prime}(t)>C(t) \text { for } t \in[\tau, \tau+\varepsilon]
$$

A path $(K(t), R(t), C(t))$ from $(K, S)$ is called efficient if it is not inefficient.

An interior path $(K(t), R(t), C(t))$ from $(K, S)$ in $\mathbb{R}_{+}^{2}$ is called competitive if it satisfies Hotelling's Rule equating the returns on the capital good and the exhaustible resource ${ }^{7}$ :

$$
\dot{F}_{2}(K(t), R(t), N(t)) / F_{2}(K(t), R(t), N(t))=F_{1}(K(t), R(t), N(t))
$$

\footnotetext{
${ }^{6}$ Note that along an interior path $(K(t), R(t), C(t))$, using $(3)(\mathrm{a}), \dot{K}(t)$ is itself a continuously differentiable function of $t$.

${ }^{7}$ It is well-known (see, for example, Dasgupta and Heal (1979)) that this rule incorporates short-run or myopic efficiency. Note that for an interior path $(K(t), R(t), C(t)), F_{2}(K(t), R(t), N(t))$ is a continuously differentiable function of $t$; this allows us to write Hotelling's rule as in (5).
} 


\subsection{Hotelling's Rule and Profit Maximization}

If $(K(t), R(t), C(t))$ is an interior path from $(K, S)$ in $\mathbb{R}_{+}^{2}$, we can associate with it a path of shadow prices $(p(t), w(t))$ as follows:

$$
\begin{aligned}
& p(t)=1 / F_{2}(K(t), R(t), N(t)) \\
& w(t)=p(t) F_{3}(K(t), R(t), N(t))
\end{aligned}
$$

Then, given the concavity of $F$, one can verify that Hotelling's Rule (5) implies profit maximization; that is, for all $t \geq 0$, and all $(K, R, N) \in \mathbb{R}_{+}^{3}$, we have:

$$
\begin{aligned}
& p(t) F(K(t), R(t), N(t))-(-\dot{p}(t)) K(t)-R(t)-w(t) N(t) \\
\geq & p(t) F(K, R, N)-(-\dot{p}(t)) K-R-w(t) N
\end{aligned}
$$

Here, $p(t)$ is the price of the capital cum consumption good, measured in units of the resource stock at time 0 , which is the numeraire, and $(-\dot{p}(t))$ is to be interpreted as the rental rate on capital. Since the resource stock does not directly affect output at any $t$ (it is only the flow of the resource which affects the output) the present value price of the resource is constant; this constant is positive, given that the production function is specified by (1). Thus, the present value price of the resource at time $t$, measured in units of the resource stock at time 0 , is unity. ${ }^{8}$ Since $F$ exhibits constant returns to scale, profit maximization implies that we also have for all $t \geq 0$,

$$
0=p(t) F(K(t), R(t), N(t))-(-\dot{p}(t)) K(t)-R(t)-w(t) N(t)
$$

\section{Properties of Competitive Paths}

We start our analysis by examining the properties of (interior) competitive paths, whether they are equitable or not. These properties will be used in the next two sections to study the nature of competitive equitable paths.

Let $(K(t), R(t), C(t))$ from $(K, S)$ in $\mathbb{R}_{+}^{2}$ be an arbitrary interior competitive path, with associated shadow prices $(p(t), w(t))$, given by (6). We show in the first subsection (Proposition 1) that such a path must satisfy the capital value transversality condition (CVTC):

$$
\lim _{t \rightarrow \infty} p(t) K(t)=0
$$

Using this property, we establish in the second subsection (Proposition 2) a value-maximizing property of the competitive path.

In the third subsection, we use the necessity of the CVTC for competitive paths to provide a characterization of competitive efficiency (Proposition 3) in terms of the CVTC and a resource exhaustion condition (REC). Using this characterization, we also provide in the fourth subsection (Proposition 4) a characterization of efficient paths in terms of the competitive condition (5), the CVTC and the REC, by noting that an efficient path is necessarily competitive. The results of the third and fourth subsections are not directly relevant for the material in the rest of the paper, since (long-run) efficiency does not a play a crucial role in the results we develop there. However, they provide the connection between competition and efficiency, which was established in Mitra (1978b) for a discrete-time exhaustible resource model, and appears to be missing for the continuous-time counterpart.

\footnotetext{
${ }^{8}$ See Mitra (1978b) for a formal proof.
} 


\subsection{The Capital Value Transversality Condition}

Proposition 1 Let $(K(t), R(t), C(t))$ from $(K, S)$ in $\mathbb{R}_{+}^{2}$ be an arbitrary interior competitive path, with associated shadow prices $(p(t), w(t))$, given by (6). Then, the capital value transversality condition:

$$
\lim _{t \rightarrow \infty} p(t) K(t)=0
$$

must hold.

Proof. Using 3(a) and (8), we have for all $t \geq 0$ :

$$
\begin{aligned}
p(t) C(t) & =p(t) F(K(t), R(t), N(t))-p(t) \dot{K}(t) \\
& =R(t)+w(t) N(t)-[\dot{p}(t)) K(t)+p(t) \dot{K}(t)]
\end{aligned}
$$

Denoting $p(t) K(t)$ by $V(t)$ for $t \geq 0$, we get:

$$
p(t) C(t)=R(t)+w(t) N(t)-\dot{V}(t)
$$

So, integrating from 0 to $T>0$, we obtain:

$$
\int_{0}^{T} p(t) C(t) d t=\int_{0}^{T} R(t) d t+\int_{0}^{T} w(t) N(t) d t-(V(T)-V(0))
$$

Note that for all $T>0$, we have:

$$
P(T) \equiv \int_{0}^{T} R(t) d t \leq S
$$

Since $P(T)$ is monotonically non-decreasing in $T$, and bounded above by $S$, it must converge. Using (6), we have for $t \geq 0$ :

$$
\begin{aligned}
w(t) N(t) & =p(t) F_{3}(K(t), R(t), N(t)) N(t) \\
& =p(t)(1-\alpha-\beta) F(K(t), R(t), N(t)) \\
& =\frac{(1-\alpha-\beta)}{\beta} p(t) F_{2}(K(t), R(t), N(t)) R(t) \\
& =\frac{(1-\alpha-\beta)}{\beta} R(t)
\end{aligned}
$$

Using (13), we obtain:

$$
W(T) \equiv \int_{0}^{T} w(t) N(t) d t \leq[(1-\alpha-\beta) / \beta] S
$$

Since $W(T)$ is monotonically non-decreasing in $T$, and bounded above (by (14)), it must converge.

Using (13) in (12), we get:

$$
\begin{aligned}
U(T) & \equiv \int_{0}^{T} p(t) C(t) d t \\
& \leq \int_{0}^{T} R(t) d t+\int_{0}^{T} w(t) N(t) d t+p(0) K(0) \\
& \leq[(1-\alpha) / \beta] \int_{0}^{T} R(t) d t+p(0) K(0) \\
& \leq[(1-\alpha) / \beta] S+p(0) K
\end{aligned}
$$


Since $U(T)$ is monotonically non-decreasing in $T$, and bounded above (by (15)), it must converge.

Returning now to (12), we note that since $P(T), W(T)$ and $U(T)$ converge as $T \rightarrow \infty$, we must also have $V(T)$ converging as $T \rightarrow \infty$. Denote the limit of $V(T)$, as $T \rightarrow \infty$, by $\nu$. We claim that $\nu=0$. Suppose, on the contrary, that $\nu>0$.

Using the definition of $p(t)$, we see that:

$$
\begin{aligned}
V(t) & =p(t) K(t)=\frac{R(t) K(t)}{R(t) F_{2}(K(t), R(t), N(t))} \\
& =\frac{R(t) K(t)}{\beta F(K(t), R(t), N(t))} \\
& =\frac{\alpha R(t) K(t)}{\beta K(t) F_{1}(K(t), R(t), N(t))} \\
& =\frac{(\alpha / \beta) R(t)}{F_{1}(K(t), R(t), N(t))}
\end{aligned}
$$

Since $\lim _{t \rightarrow \infty} V(t)=\nu$, there is $T^{\prime}>0$, such that for all $t \geq T^{\prime}, V(t) \geq(\nu / 2)$, and using this in (16), we obtain:

$$
F_{1}(K(t), R(t), N(t)) \leq(2 / \nu)(\alpha / \beta) R(t) \text { for all } t \geq T^{\prime}
$$

Thus, we have, for all $T>T^{\prime}$,

$$
\begin{aligned}
\int_{0}^{T} F_{1}(K(t), R(t), N(t)) d t= & \int_{0}^{T^{\prime}} F_{1}(K(t), R(t), N(t)) d t \\
& +\int_{T^{\prime}}^{T} F_{1}(K(t), R(t), N(t)) d t \\
\leq & M T^{\prime}+(2 / \nu)(\alpha / \beta) S \equiv \theta
\end{aligned}
$$

where $M$ is the maximum value of the continuous function $F_{1}(K(t), R(t), N(t))$ on the interval $\left[0, T^{\prime}\right]$.

Using (5) and (6), we have for all $t \geq 0$,

$$
\dot{p}(t) / p(t)=-F_{1}(K(t), R(t), N(t))
$$

Thus, for all $T>0$, by integrating (19), we obtain

$$
\ln p(0)-\ln p(T)=\int_{0}^{T} F_{1}(K(t), R(t), N(t)) d t
$$

Using (18) and (20), we obtain for all $T>0$ :

$$
\ln p(T) \geq \ln p(0)-\theta
$$

so that $p(t)$ is bounded away from zero. Since $p(t)$ decreases with $t$, it must converge to a positive number, say $\pi$, as $t \rightarrow \infty$. Since $p(t) K(t)$ converges to $\nu$, we must have $K(t)$ converging to $(\nu / \pi)$ as $t \rightarrow \infty$. Using (6) again, we have :

$$
(1 / p(t))=\frac{K(t)^{\alpha} N(t)^{(1-\alpha-\beta)}}{R(t)^{1-\beta}} \text { for } t \geq 0
$$


and so we obtain, for $t \geq 0$ :

$$
R(t)^{1-\beta}=p(t) K(t)^{\alpha} N(t)^{(1-\alpha-\beta)} \geq p(t) K(t)^{\alpha} N(0)^{(1-\alpha-\beta)}
$$

Since $p(t)$ converges to $\pi$, and $K(t)$ converges to $(\nu / \pi)$ as $t \rightarrow \infty$, there is some $T^{\prime \prime}$, such that for all $t \geq T^{\prime \prime}$, we have:

$$
R(t)^{1-\beta} \geq p(t) K(t)^{\alpha} N(0)^{(1-\alpha-\beta)} \geq\left(\frac{1}{2}\right) \pi(\nu / \pi)^{\alpha} N(0)^{(1-\alpha-\beta)}
$$

But this violates the resource constraint, (3)(b). This contradiction establishes our claim that $\nu=0$, and the capital value transversality condition is proved.

\subsection{The Value Maximizing Property}

Proposition 2 Let $(K(t), R(t), C(t))$ from $(K, S)$ in $\mathbb{R}_{+}^{2}$ be an arbitrary interior competitive path, with associated shadow prices $(p(t), w(t))$, given by (6). Then, it satisfies the following value-maximizing property:

$$
\begin{aligned}
0= & \int_{T}^{\infty} p(t) C(t) d t-\int_{T}^{\infty} w(t) N(t) d t-p(T) K(T)-E(T) \geq \\
& \int_{T}^{\infty} p(t) C(t+\tau) d t-\int_{T}^{\infty} w(t) N(t+\tau) d t \\
& -p(T) K(T+\tau))-E(T+\tau)
\end{aligned}
$$

holds for all $T>0$, and all $\tau$, satisfying $0<|\tau|<T$.

Proof. Using (12) and the capital value transversality condition, and noting that the three integrals in (12) converge as $T \rightarrow \infty$, we get:

$$
\int_{0}^{\infty} p(t) C(t) d t=\int_{0}^{\infty} R(t) d t+\int_{0}^{\infty} w(t) N(t) d t+p(0) K(0)
$$

The relationship noted in (22) can be generalized as follows. Pick any $T \geq 0$. Using (11), and integrating from $T$ to $T^{\prime}>T$, we obtain:

$$
\int_{T}^{T^{\prime}} p(t) C(t) d t=\int_{T}^{T^{\prime}} R(t) d t+\int_{T}^{T^{\prime}} w(t) N(t) d t-\left(V\left(T^{\prime}\right)-V(T)\right)
$$

Letting $T^{\prime} \rightarrow \infty$, using the transversality condition, and noting that the three integrals in (23) converge as $T^{\prime} \rightarrow \infty$, we obtain:

$$
\int_{T}^{\infty} p(t) C(t) d t=\int_{T}^{\infty} R(t) d t+\int_{T}^{\infty} w(t) N(t) d t+p(T) K(T) \text { for all } T \geq 0
$$

Denote $\int_{0}^{\infty} R(t) d t$ by $E$, and for each $T \geq 0$, define:

$$
E(T)=E-\int_{0}^{T} R(t) d t
$$


Clearly, $E(0)=E, E(T) \geq 0$ for $T \geq 0$, and $E(T) \rightarrow 0$ as $T \rightarrow \infty$. Further, we have, for $T \geq 0$,

$$
E(T)=\int_{T}^{\infty} R(t) d t
$$

Using (25) in (24), we obtain, for each $T \geq 0$,

$$
\int_{T}^{\infty} p(t) C(t) d t-\int_{T}^{\infty} w(t) N(t) d t-p(T) K(T)-E(T)=0 \text { for all } T \geq 0
$$

Let $T>0$ and $|\tau| \in(0, T)$ be given. Note that for each $t \geq T$, using (7) and (8), we have, for all $(K, R, N) \in \mathbb{R}_{+}^{3}$ :

$$
p(t) F(K, R, N)-(-\dot{p}(t)) K-R-w(t) N \leq 0
$$

Then, using (27) on the triple $(K(t+\tau), R(t+\tau), N(t+\tau))$, we have:

$$
\begin{gathered}
p(t) F(K(t+\tau), R(t+\tau), N(t+\tau))-(-\dot{p}(t)) K(t+\tau) \\
-R(t+\tau)-w(t) N(t+\tau) \leq 0
\end{gathered}
$$

and using the fact that $F(K(t+\tau), R(t+\tau), N(t+\tau))=C(t+\tau)+\dot{K}(t+\tau)$ in (28), we obtain:

$$
\begin{aligned}
p(t) C(t+\tau) & =p(t) F(K(t+\tau), R(t+\tau), N(t+\tau))-p(t) \dot{K}(t+\tau) \\
& \leq R(t+\tau)+w(t) N(t+\tau)-d[p(t)) K(t+\tau)] / d t
\end{aligned}
$$

Integrating (29) from $T$ to $T^{\prime}>T$, we get:

$$
\begin{aligned}
\int_{T}^{T^{\prime}} p(t) C(t+\tau) d t \leq & \int_{T}^{T^{\prime}} R(t+\tau) d t+\int_{T}^{T^{\prime}} w(t) N(t+\tau) d t \\
& -\left[p\left(T^{\prime}\right) K\left(T^{\prime}+\tau\right)-p(T) K(T+\tau)\right] \\
\leq & \int_{T}^{T^{\prime}} R(t+\tau) d t+\int_{T}^{T^{\prime}} w(t) N(t+\tau) d t \\
& +p(T) K(T+\tau))
\end{aligned}
$$

Using the fact that $g(t) \leq g$ for $t \geq T$, it is easy to check that:

$$
\frac{N(t+\tau)}{N(t)} \leq e^{g|\tau|} \text { for all } t \geq T
$$

This implies that:

$$
w(t) N(t+\tau) \leq w(t) N(t) e^{g|\tau|} \text { for all } t \geq T
$$

Using (31), (3)(b) and (14), it follows that the three integrals in (30) must converge as $T^{\prime} \rightarrow \infty$, and we get:

$$
\begin{aligned}
\int_{T}^{\infty} p(t) C(t+\tau) d t & \left.\leq \int_{T}^{\infty} R(t+\tau) d t+\int_{T}^{\infty} w(t) N(t+\tau) d t+p(T) K(T+\tau)\right) \\
& \left.=\int_{T+\tau}^{\infty} R(s) d s+\int_{T}^{\infty} w(t) N(t+\tau) d t+p(T) K(T+\tau)\right) \\
& \left.=E(T+\tau)+\int_{T}^{\infty} w(t) N(t+\tau) d t+p(T) K(T+\tau)\right)
\end{aligned}
$$


Using (26) and (32), we obtain:

$$
\begin{aligned}
0= & \int_{T}^{\infty} p(t) C(t) d t-\int_{T}^{\infty} w(t) N(t) d t-p(T) K(T)-E(T) \geq \\
& \int_{T}^{\infty} p(t) C(t+\tau) d t-\int_{T}^{\infty} w(t) N(t+\tau) d t \\
& -p(T) K(T+\tau))-E(T+\tau)
\end{aligned}
$$

as claimed in the proposition.

\subsection{Efficiency of Competitive Paths}

Proposition 3 Let $(K(t), R(t), C(t))$ from $(K, S)$ in $\mathbb{R}_{+}^{2}$ be an arbitrary interior competitive path, with associated shadow prices $(p(t), w(t))$, given by $(6)$. Then, $(K(t), R(t), C(t))$ is efficient from $(K, S)$ if and only if it satisfies:

$$
\begin{aligned}
& \text { (i) } \lim _{t \rightarrow \infty} p(t) K(t)=0 \\
& \text { (ii) } \int_{0}^{\infty} R(t) d t=S
\end{aligned}
$$

Proof. (Necessity) Let $(K(t), R(t), C(t))$ from $(K, S)$ in $\mathbb{R}_{+}^{2}$ be an arbitrary interior competitive path, with associated shadow prices $(p(t), w(t))$, given by $(6)$. Then $(i)$ follows from Proposition 1. If $(K(t), R(t), C(t))$ is efficient from $(K, S)$, we establish (ii) by supposing that it is violated. Then, by $(3)(\mathrm{b})$, we have:

$$
e \equiv S-\int_{0}^{\infty} R(t) d t>0
$$

Define a function $(e(t))$ as follows:

$$
e(t)= \begin{cases}3 e(1-t)^{2} & \text { for } t \in[0,1] \\ 0 & \text { for } t>1\end{cases}
$$

Then, $e(t)$ is a continuously differentiable function of $t$ on $[0, \infty)$, and:

$$
\int_{0}^{\infty} e(t) d t=e
$$

Define $\left(K^{\prime}(t), R^{\prime}(t), C^{\prime}(t)\right)$ as follows:

$$
\left.\begin{array}{ll}
K^{\prime}(t)=K(t) & \text { for } t \geq 0 \\
R^{\prime}(t)=R(t)+e(t) & \text { for } t \geq 0 \\
C^{\prime}(t)=F\left(K^{\prime}(t), R^{\prime}(t), N(t)\right)-\dot{K}^{\prime}(t) & \text { for } t \geq 0
\end{array}\right\}
$$

Then, it is easy to check that $\left(K^{\prime}(t), R^{\prime}(t), C^{\prime}(t)\right)$ is a path from $(K, S)$. Since $R^{\prime}(t) \geq R(t)$ for all $t$, and $K^{\prime}(t)=K(t)$ for all $t$, we must have $C^{\prime}(t) \geq C(t)$ for all $t$. Further, since $K>0$, there is $\tau \in(0,1)$, such that for $t \in[0, \tau], K(t)>0$. Since we have $R^{\prime}(t)>R(t)$ for all $t \in[0, \tau]$, we must have $C^{\prime}(t)>C(t)$ for all $t \in[0, \tau]$. This means that $(K(t), R(t), C(t))$ must be inefficient, a contradiction which establishes (ii).

(Sufficiency) This is standard, and can be established by following the proof of Lemma 6 in Asheim, Buchholz, Hartwick, Mitra and Withagen (2006). 


\subsection{A Characterization of Efficiency}

Proposition 4 Let $(K(t), R(t), C(t))$ from $\left(K_{o}, S_{o}\right)$ in $\mathbb{R}_{+}^{2}$ be an arbitrary interior path. Then, $(K(t), R(t), C(t))$ is efficient from $\left(K_{o}, S_{o}\right)$ if and only if it satisfies Hotelling's rule (given by (5)), and the following two conditions:

$$
\begin{aligned}
& \text { (i) } \lim _{t \rightarrow \infty}\left[1 / F_{2}(K(t), R(t), N(t))\right] K(t)=0 \\
& \text { (ii) } \int_{0}^{\infty} R(t) d t=S_{o}
\end{aligned}
$$

Proof. (Sufficiency) Since $(K(t), R(t), C(t))$ from $\left(K_{o}, S_{o}\right)$ in $\mathbb{R}_{+}^{2}$ is an interior competitive path, we can associate with it shadow prices $(p(t), w(t))$, given by $(6)$. Since (i) holds we have $\lim _{t \rightarrow \infty} p(t) K(t)=0$. And, since (ii) also holds, the path $(K(t), R(t), C(t))$ is efficient by Proposition 3.

(Necessity) We establish that if $(K(t), R(t), C(t))$ from $\left(K_{o}, S_{o}\right)$ is efficient, then it satisfies (5). Using the necessity proof of Proposition 4 , it is easy to check that $(K(t), S(t))$ solves the problem:

$$
\left.\begin{array}{ll}
\text { Max } & \int_{0}^{\infty} \dot{S}^{\prime}(t) d t \\
\text { subject to } & F\left(K^{\prime}(t),-\dot{S}^{\prime}(t), N(t)\right)-\dot{K}(t) \geq C(t) \text { for } t \geq 0 \\
& -\dot{S}^{\prime}(t) \geq 0 \text { for } t \geq 0 \\
& S^{\prime}(t), K^{\prime}(t) \geq 0 \text { for } t \geq 0 \\
& S^{\prime}(0), K^{\prime}(0)=\left(S_{o}, K_{o}\right)
\end{array}\right\}(P)
$$

Notice that $(P)$ is in the standard format of an infinite-horizon optimal control problem (with time-dependent feasibility sets). We will invoke the maximum principle for this problem.

Define $Y(t)=\{(K, S),(Z, W): F(K,-W, N(t))-Z \geq C(t),(K, S) \geq 0,-W \geq 0\}$, and $u: Y(t) \times \mathbb{R}_{+} \rightarrow \mathbb{R}$ by $u((K, S),(Z, W), t)=W$. Then, $Y(t)$ and $u$ satisfy assumptions (A.1)(A.3) of Takekuma (1982). And, since $(K(t), R(t), C(t))$ is interior, (A.4) is also satisfied. We can therefore apply his "Main Theorem" (p.431) and Remark 4.2 (p.432).

To this end, define:

$$
\begin{aligned}
H((\pi, \eta),(K, S), t) & =\sup \{u((K, S),(Z, W), t)+\pi Z+\eta W: \\
((K, S),(Z, W)) & \in Y(t)\}
\end{aligned}
$$

Then, we have absolutely continuous functions $\{\pi(t), \eta(t)\}$ with $\pi(t)>0$ for $t \geq 0$, such that:

$$
\left.\begin{array}{l}
(i) d(K(t), S(t)) / d t \in \partial_{1} H((\pi(t), \eta(t)),(K(t), S(t)), t) \\
(i i)-(\dot{\pi}(t), \dot{\eta}(t)) \in \partial_{2} H((\pi(t), \eta(t)),(K(t), S(t)), t) \\
(i i i) H((\pi(t), \eta(t)),(K(t), S(t)), t)=d S(t) / d t+\pi(t) d K(t) / d t+\eta(t) d S(t) / d t
\end{array}\right\}
$$

It follows from (ii) that:

$$
\left.\begin{array}{l}
-\dot{\pi}(t)=\pi(t) F_{1}(K(t), R(t), N(t)) \\
-\dot{\eta}(t)=0
\end{array}\right\}
$$

Furthermore, it follows from (iii) that:

$$
1-\pi(t) F_{2}(K(t), R(t), N(t))=\eta(t)
$$


Differentiating this equation with respect to $t$, and using $\dot{\eta}(t)=0$, we get:

$$
\pi(t) \dot{F}_{2}(K(t), R(t), N(t))+\dot{\pi}(t) F_{2}(K(t), R(t), N(t))=0
$$

Using (33) now yields:

$$
\pi(t)\left[\dot{F}_{2}(K(t), R(t), N(t))-F_{2}(K(t), R(t), N(t)) F_{1}(K(t), R(t), N(t))\right]=0
$$

Since $\pi(t)>0$ for $t \geq 0$, (5) must hold for all $t \geq 0$.

Conditions (i) and (ii) now follow directly from Proposition 3.

\section{Competitive Equity and a Generalized Hartwick Rule}

In the context of our model (but for a more general class of production functions than (1)), in which population is stationary, Hartwick (1977) made the interesting observation that a competitive path, which follows the rule of investing the rents from the exhaustible resource used at each date, in the net accumulation of the produced capital good, is equitable. We shall refer to this investment rule as Hartwick's rule. As Solow (1986) has observed, this is an intuitively appealing investment rule of maintaining the consumption potential of society, in a generalized sense, by replacing exhaustible resource stocks, which are used up, with produced capital goods of equal value.

It turns out that Hartwick's Rule has significance in a wider class of models than the special context in which it arose initially. In particular, Dixit, Hammond and Hoel (1980) recognized that Hartwick's Rule is really a statement that the valuation of net investment (including the dis-investment in the exhaustible resource) is zero at each date. They then proceeded to show in a general model of accumulation involving heterogenous capital goods (which could include various non-renewable resource stocks) that if the valuation of net investment is constant over time (the constant is not required to be zero) then this would ensure intertemporal equity (in the sense described above, but with "consumption" interpreted now as the utility based on a vector of consumption goods). Furthermore, this investment rule, which might legitimately be called the Dixit-Hammond-Hoel rule was also a necessary condition for intertemporal equity along competitive paths.

This characterization of competitive equitable paths naturally leads one to re-examine the special significance of Hartwick's rule for intertemporal equity. This issue was first examined by Dasgupta and Mitra (1983); however, their investgation was in the context of a discrete-time model, where Hartwick's rule does not hold in the original form but rather in a modified form, for efficient equitable paths. In the corresponding continuous time framework, Hartwick's rule does hold in its original form as a necessary condition along efficient equitable paths; see, for example, Withagen and Asheim (1998) and Mitra (2002)for versions of this result in general models of accumulation involving heterogenous capital goods (which could include various non-renewable resource stocks) ${ }^{9}$.

Buchholz, Dasgupta and Mitra (2005) showed that, in the context of the exhaustible resource model in which Hartwick first proposed his rule, Hartwick's rule is necessary for

\footnotetext{
${ }^{9}$ Our review of this literature is deliberately brief, since there is a comprehensive appraisal of this line of research in Asheim, Buchholz and Withagen (2003). In particular, their paper explores the relation between Hartwick's rule and the theory of sustainability.
} 
intertemporal equity of competitive paths, provided the exhaustible resource is "important" in production. In particular, this result holds when the production function is specified by (1). That is, in the context of our model, but without population growth, if a path $(K(t), R(t), C(t))$ from $(K, S)$ in $\mathbb{R}_{+}^{2}$ is interior, competitive and equitable, then the value of net investment, in both the produced capital good, and the non-produced capital good (the exhaustible resource), $I(t) \equiv p(t) \dot{K}(t)+\dot{S}(t)$, must equal zero at each date. In contrast to the literature mentioned in the previous paragraph, the rather demanding assumption of efficiency of these paths is irrelevant in this particular context. This result implies the rather intriguing fact that in the context of this model, competitive paths which satisfy the Dixit-Hammond-Hoel rule (that the value of net investment be constant) must also satisfy Hartwick's rule (that the value of net investment be zero).

In the case of our framework of Section 2, which allows for a growing population (as well as a stationary population), we establish in this section the following generalization of the result indicated in the previous paragraph. If a path $(K(t), R(t), C(t))$ from $(K, S)$ in $\mathbb{R}_{+}^{2}$ is interior, competitive and equitable, then the value of net investment must change according to the formula:

$$
\dot{I}(t)=[w(t) N(t)-p(t) C(t)] g(t) \text { for all } t \geq 0
$$

This formula reduces to Hartwick's rule in the stationary population case (that is, when $g(t)=0$ for $t \geq 0$ ), and therefore represents an appropriate generalization of Hartwick's rule.

Theorem 1 Let $(K(t), R(t), C(t))$ from $(K, S)$ in $\mathbb{R}_{+}^{2}$ be an arbitrary interior competitive equitable path, with associated shadow prices $(p(t), w(t))$, given by $(6)$, and with constant per-capita consumption $(C(t) / N(t))=c>0$ for $t \geq 0$. Then, it must satisfy:

$$
\begin{aligned}
& p(T) \dot{K}(T)-R(T) \\
= & \int_{T}^{\infty} p(t) c \dot{N}(t) d t-\int_{T}^{\infty} w(t) \dot{N}(t) d t
\end{aligned}
$$

for all $T \geq 0$, and:

$$
\dot{I}(t)=[w(t) N(t)-p(t) C(t)] g(t) \text { for all } t \geq 0
$$

Proof. The idea of the proof (of the first formula) is to use the value maximizing property established in Proposition 2, and to write down the necessary first-order condition. Note that since the value-maximization property involves time-shifted primal variables, but not the dual variables, time derivatives of only the primal variables will appear. However, in order to do this, one has to show that the interchange of the integral operation and the limit operation is justified. Thus, a "dominated convergence theorem" for Riemann integrals has to be used. The bulk of the proof below is in making sure of this important step. The second formula follows directly from the first.

Fix an arbitrary $T>0$, and let $0<\tau<T$. Then, using the value-maximizing property established in Proposition 2, we get:

$$
\begin{aligned}
& p(T)(K(T+\tau)-K(T))+E(T+\tau)-E(T) \\
\geq & \int_{T}^{\infty} p(t)(C(t+\tau)-C(t)) d t-\int_{T}^{\infty} w(t)(N(t+\tau)-N(t)) d t
\end{aligned}
$$


Further, by dividing through by $\tau>0$, we obtain:

$$
\begin{aligned}
& p(T) \frac{(K(T+\tau)-K(T))}{\tau}+\frac{E(T+\tau)-E(T)}{\tau} \\
\geq & \int_{T}^{\infty} p(t) \frac{(C(t+\tau)-C(t))}{\tau} d t-\int_{T}^{\infty} w(t) \frac{(N(t+\tau)-N(t))}{\tau} d t
\end{aligned}
$$

We claim that:

$$
\begin{aligned}
& p(T) \dot{K}(T)+\dot{E}(T) \\
\geq & \int_{T}^{\infty} p(t) c \dot{N}(t) d t-\int_{T}^{\infty} w(t) \dot{N}(t) d t
\end{aligned}
$$

Pick $N \in \mathbb{N}$ such that $(1 / N)<T$. then, for each $n \in \mathbb{M}=\{n \in \mathbb{N}$, with $n \geq N\}$ we have $(1 / n) \in(0, T)$. Thus, for each $n \in \mathbb{M}$, we can write, using $(34)$,

$$
\begin{aligned}
& p(T) \frac{(K(T+(1 / n))-K(T))}{(1 / n)}+\frac{E(T+(1 / n))-E(T)}{(1 / n)} \\
\geq & \int_{T}^{\infty} p(t) \frac{(C(t+(1 / n))-C(t))}{(1 / n)} d t \\
& -\int_{T}^{\infty} w(t) \frac{(N(t+(1 / n))-N(t))}{(1 / n)} d t
\end{aligned}
$$

Letting $n \rightarrow \infty$, we see that the left-hand side of (36) clearly converges to the left hand side of (35), since the derivatives $\dot{K}(T)$ and $\dot{E}(T)$ exist. For the right hand side expressions in (36) and (35), one needs some more work to validate the claim.

We analyze, first, the second integral appearing in the right hand side of (36). Let $\varepsilon>0$ be given. Note that:

$$
w(t) \dot{N}(t) \leq g w(t) N(t) \text { for all } t \geq 0
$$

and so for $B>T$ :

$$
\int_{T}^{B} w(t) \dot{N}(t) d t \leq g \int_{T}^{B} w(t) N(t) d t \leq g[(1-\alpha-\beta) / \beta] S
$$

Since $\int_{T}^{B} w(t) \dot{N}(t) d t$ is monotonically non-decreasing in $B>T$, it converges as $B \rightarrow \infty$. This limit is denoted by $\int_{T}^{\infty} w(t) \dot{N}(t) d t$, an improper Riemann integral. Clearly, one can pick $T^{\prime}>T$, such that:

$$
\int_{T}^{\infty} w(t) \dot{N}(t) d t-\int_{T}^{T^{\prime}} w(t) \dot{N}(t) d t<\varepsilon / 3
$$

Similarly, for all $n \in \mathbb{M}$, we have:

$$
w(t) \frac{(N(t+(1 / n))-N(t))}{(1 / n)} \leq g e^{g T} w(t) N(t) \text { for all } t \geq 0
$$

For $B>T$ :

$$
\int_{T}^{B} g e^{g T} w(t) N(t) d t \leq g e^{g T}[(1-\alpha-\beta) / \beta] S
$$


Since $\int_{T}^{B} g e^{g T} w(t) N(t) d t$ is monotonically non-decreasing in $B>T$, it converges as $B \rightarrow \infty$. This limit is denoted by $\int_{T}^{\infty} g e^{g T} w(t) N(t) d t$, an improper Riemann integral. Clearly, one can pick $T^{\prime \prime}>T$, such that $\int_{T^{\prime \prime}}^{\infty} g e^{g T} w(t) N(t) d t<\varepsilon / 3$. Then, for every $n \in \mathbb{M}$, the improper Riemann integral $\int_{T}^{\infty} w(t)\left[\frac{(N(t+(1 / n))-N(t))}{(1 / n)}\right] d t$ exists, and one can pick $T^{\prime \prime}>T^{\prime}$, such that

$$
\int_{T}^{\infty} w(t) \frac{(N(t+(1 / n))-N(t))}{(1 / n)} d t-\int_{T}^{T^{\prime \prime}} w(t) \frac{(N(t+(1 / n))-N(t))}{(1 / n)} d t<\varepsilon / 3
$$

Define:

$$
\left.\begin{array}{l}
f_{n}(t)=w(t) \frac{(N(t+(1 / n))-N(t))}{(1 / n)} \text { for } t \in\left[T, T^{\prime \prime}\right], n \in \mathbb{M} \\
f(t)=w(t) \dot{N}(t) \text { for } t \in\left[T, T^{\prime \prime}\right]
\end{array}\right\}
$$

and note that, for each $t \in\left[T, T^{\prime \prime}\right]$,

$$
\lim _{n \rightarrow \infty} f_{n}(t)=f(t)
$$

by definition of a derivative. Further, for each $n \in \mathbb{M}, f_{n}$ is a continuous function on $\left[T, T^{\prime \prime}\right]$; and $f(t)=w(t) N(t) g(t)$ is also a continuous function on $\left[T, T^{\prime \prime}\right]$. Thus, each $f_{n}$ (for $n \in \mathbb{M}$ ) is Riemann integrable on $\left[T, T^{\prime \prime}\right]$, and so is $f$. Finally, each $f_{n}$ (for $n \in \mathbb{M}$ ) is non-negative, and bounded above by the function:

$$
h(t)=w(t) N(t) g e^{g T} \text { for all } t \in\left[T, T^{\prime \prime}\right]
$$

and $h$, being clearly continuous on $\left[T, T^{\prime \prime}\right]$, is also Riemann integrable on $\left[T, T^{\prime \prime}\right]$. Thus, we can conclude that:

$$
\lim _{n \rightarrow \infty} \int_{T}^{T^{\prime \prime}} f_{n}(t) d t=\int_{T}^{T^{\prime \prime}} f(t) d t
$$

See Cunningham (1967, Theorem 2, p. 184) for the precise result that is being used.

Using (41), we can choose $\mu \in \mathbb{M}$, such that:

$$
\left|\int_{T}^{T^{\prime \prime}} f_{n}(t) d t-\int_{T}^{T^{\prime \prime}} f(t) d t\right|<\varepsilon / 3 \text { for all } n \geq \mu
$$

Then, using (37), (38) and (42), we get for all $n \geq \mu$,

$$
\left|\int_{T}^{\infty} f_{n}(t) d t-\int_{T}^{\infty} f(t) d t\right|<\varepsilon
$$

This establishes that:

$$
\lim _{n \rightarrow \infty} \int_{T}^{\infty} f_{n}(t) d t=\int_{T}^{\infty} f(t) d t
$$

The analysis of the first inegral appearing in the right hand side of (36) is similar, by noting that $C(t)=c N(t)$ for $t \geq 0$. And, it leads to the result that:

$$
\lim _{n \rightarrow \infty} \int_{T}^{\infty} p(t) \frac{(C(t+(1 / n))-C(t))}{(1 / n)} d t=\int_{T}^{\infty} p(t) c \dot{N}(t) d t
$$

Using (43) and (44), one establishes the claim (35). 
If one uses the value maximizing property with $\tau<0$, but $|\tau|<T$, one can denote $(-\tau)$ by $\tau^{\prime}$, and obtain:

$$
\begin{aligned}
& p(T) \frac{\left(K(T)-K\left(T-\tau^{\prime}\right)\right)}{\tau^{\prime}}+\frac{E(T)-E\left(T-\tau^{\prime}\right)}{\tau^{\prime}} \\
\leq & \int_{T}^{\infty} p(t) \frac{\left(C(t)-C\left(t-\tau^{\prime}\right)\right)}{\tau^{\prime}} d t-\int_{T}^{\infty} w(t) \frac{\left(N(t)-N\left(t-\tau^{\prime}\right)\right)}{\tau^{\prime}} d t
\end{aligned}
$$

The preceding analysis will then establish the following claim:

$$
\begin{aligned}
& p(T) \dot{K}(T)+\dot{E}(T) \\
\leq & \int_{T}^{\infty} p(t) c \dot{N}(t) d t-\int_{T}^{\infty} w(t) \dot{N}(t) d t
\end{aligned}
$$

Combining (35) and (45), we get:

$$
\begin{aligned}
& p(T) \dot{K}(T)-R(T) \\
= & \int_{T}^{\infty} p(t) c \dot{N}(t) d t-\int_{T}^{\infty} w(t) \dot{N}(t) d t
\end{aligned}
$$

As noted earlier, $\dot{K}(t)$ is itself a differentiable function of time (by $3($ a)). So, we can denote the value of net investment, $p(T) \dot{K}(T)-R(T)$, by $I(T)$, and use (46) to obtain:

$$
\begin{aligned}
\dot{I}(T) & =w(T) \dot{N}(T)-p(T) c \dot{N}(T) \\
& =[w(T) N(T)-p(T) C(T)] g(T)
\end{aligned}
$$

\section{Parametric Restrictions for Competitive Equity}

We now establish certain restrictions on the framework that must be satisfied for the existence of a competitive equitable path. More generally, these parametric restrictions follow from the existence of a competitive path, for which per capita consumption is bounded away from zero.

In the framework of our model, but without population growth, Solow (1974) obtained the parametric restriction for competitive equity that the capital coefficient $(\alpha)$ exceed the resource coefficient $(\beta)$ in the production function, given by $(1) .{ }^{10}$

In the current framework, which allows for growing (as well as stationary) population, the restrictions on population growth for the existence of competitive equitable path is a question of considerable interest. In a discrete-time version of the current framework, Mitra (1983) provided a restriction on the population sequence, which was necessary for the existence of an efficient equitable path; together with Solow's technological parametric restriction, a

\footnotetext{
${ }^{10}$ This result was extended to a more general class of production functions than (1), by focusing on a condition on the behavior of the "area under the isoquant", as the output level goes to zero. This class of results was independently obtained by Mitra (1978a), Buchholz (1982), and Shimomura (1983). For an account of this line of enquiry, see Kemp, Long and Shimomura (1984). The most general result along this line is contained in the paper by Cass and Mitra (1991).
} 
slightly stronger restriction was also shown to be sufficient for the existence of such a path. It can be checked that these results can also be phrased in terms of restrictions for the existence of a competitive equitable path, efficiency being not crucial to deriving these restrictions.

The general restriction on the population sequence, obtained by Mitra (1983), is somewhat hard to interpret. As an aid to understanding the restriction, it was pointed out in his paper that if the population sequence experienced quasi-arithmetic growth, with exponent $\mu \geq 0$ (and parameters $a, b>0$ ),

$$
N(t)=a+b t^{\mu} \text { for all } t \geq 0
$$

then the restriction on the population sequence amounted to the simple condition:

$$
\mu<(\alpha / \beta)-1
$$

For the case of stationary population $(\mu=0)$, this was precisely Solow's technological restriction.

In re-examining Mitra's 1983 results recently, in a continuous-time setting, Asheim, Buchholz, Hartwick, Mitra and Withagen (2006) established that if there exists a competitive equitable path with a constant savings rate, then population must follow precisely quasiarithmetic growth.

Since a constant savings rate is a somewhat ad hoc condition, the question arises whether, without that condition, quasi-arithmetic growth is, in some sense, the right restriction on population growth imposed by the existence of a competitive equitable path.

Global population change since 1990 appears to have the following stylized features: (i) Population continues to grow, and the growth is significant; (ii) the growth rate of population is steadily decreasing; (iii) the absolute increase in population is also decreasing. Feature (i) challenges the zero population growth idealization, while feature (ii) suggests that exponential growth formulation is unrealistic. Consistent with features (i) and (ii) is the abstraction, postulated in quasi-arithmetic growth. Feature (iii) suggests that population is experiencing sub-arithmetic growth.

The principal result of this section is that if there exists a competitive equitable path, then population can experience at most quasi-arithmetic growth. Further, the exponent $\mu$ (in this upper bound) can be precisely specified in terms of the technological coefficients, $\alpha$ and $\beta$.

This result is accomplished by combining several implications of competitive equity, each of which is of independent interest. The first implication is that there must be ongoing capital accumulation all along the path. Thus, even though investment is not restricted to be irreversible, it is so along competitive equitable paths. The second implication is that exhaustible resource constraints impose upper bounds on the rate at which capital accumulation can proceed. The third implication is that these upper bounds on capital accumulation, when combined with intertemporal equity, impose the Solow restrictions on the technological parameters of the model.

\subsection{The Necessity of Ongoing Capital Accumulation}

Lemma 1 Let $(K(t), R(t), C(t))$ from $(K, S)$ in $\mathbb{R}_{+}^{2}$ be an arbitrary interior competitive equitable path, with associated shadow prices $(p(t), w(t))$, given by $(6)$, and with constant 
per-capita consumption $(C(t) / N(t))=c>0$ for $t \geq 0$. Then, it must satisfy:

$$
\dot{K}(t) \geq 0 \text { for all } t \geq 0
$$

Proof. Suppose, contrary to (48), there is some $T$, for which $C(T)>Q(T)$. Denoting $Q(T)-C(T)$ by $\varepsilon$, we have $\varepsilon>0$, and:

$$
\dot{K}(T)=-\varepsilon
$$

Then, by continuity of $\dot{K}(t)$, we have $\delta>0$, such that:

$$
\dot{K}(t)<0 \text { for all } t \in[T, T+\delta]
$$

Thus, $K(t)<K(T)$ for all $t \in(T, T+\delta]$.

We claim that $K(t)<K(T)$ for all $t>T$. For, if there is some $t^{\prime}>T$, such that $K\left(t^{\prime}\right) \geq K(T)$, then $t^{\prime}>T+\delta$, and by continuity of $K(t)$, we have $\tau=\min \left\{t^{\prime}>T+\delta\right.$ : $\left.K\left(t^{\prime}\right) \geq K(T)\right\}$ well-defined, and $\tau>T+\delta$. Then,

$$
K(\tau)=K(T) \text {, and } K(t)<K(T) \text { for all } t \in(T, \tau)
$$

We focus now on $t \in(T, \tau)$. For all such $t$, we have $F_{2}(K(t), R(t), N(t))$ increasing in $t$; that is, $Q(t) / R(t)$ is increasing in $t$. This means that $[Q(t) / N(t)] /[R(t) / N(t)]$ is increasing in $t$, so that $\left[k(t)^{\alpha} r(t)^{\beta} / r(t)\right]$ must be increasing in $t$, where $k(t)=K(t) / N(t)$ and $r(t)=$ $R(t) / N(t)$ for all $t$. Using (51) and the fact that $N(t)$ is non-decreasing in $t$, we must have $k(t)<k(T)$ for all $t \in(T, \tau)$. But, then $r(t)<r(T)$ for all $t \in(T, \tau)$. Thus, for all $t \in(T, \tau)$, we get:

$$
\begin{aligned}
\dot{K}(t) / N(t) & =k(t)^{\alpha} r(t)^{\beta}-c \\
& <k(T)^{\alpha} r(T)^{\beta}-c \\
& =\dot{K}(T) / N(T)
\end{aligned}
$$

That is, using (49) and the fact that $N(t)$ is non-decreasing in $t$, we have:

$$
\dot{K}(t)<-\varepsilon \text { for all } t \in(T, \tau)
$$

This clearly implies that $K(\tau)<K(T)-\varepsilon(\tau-T)<K(T)$, a contradiction to (51). Thus, $K(t)<K(T)$ for all $t>T$ must hold. Repeating the above argument (but this time for all $t>T$ ), we have $\dot{K}(t)<-\varepsilon$ for all $t>T$. But, this means that $K(t)<0$ for large $t$, a contradiction, which establishes (48).

\subsection{An Upper Bound on Capital Accumulation}

The upper bound on capital accumulation is obtained by applying methods used in Solow (1974) and Mitra (1983). In the latter, the objective was to obtain restrictions on the path of $N(t)$ for competitive equitable paths to exist, which would be "almost" sufficient as well. Thus, there was an explicit attempt to obtain the upper bound on capital accumulation solely in terms of the path of $N(t)$. Here we use a variation, in which an upper bound is obtained in terms of the path of $N(t)$, and $t$ itself. This type of restriction is useful in obtaining the quasi-arithmetic growth restriction on population growth, as we will see later in this section. Needless to say, these methods rely heavily on the technique introduced in Solow (1974, p. 43, Appendix B), and also on the fact that the production function is of the Cobb-Douglas form. 
Lemma 2 Let $(K(t), R(t), C(t))$ from $(K, S)$ in $\mathbb{R}_{+}^{2}$ be an arbitrary interior path. Then, there is a positive constant, $A$, such that:

$$
K(t) \leq A t^{(1-\beta) /(1-\alpha)} N(t) \text { for all } t \geq 0
$$

Proof. Clearly, for each $t \geq 0$, we have:

$$
\dot{K}(t)=K(t)^{\alpha} R(t)^{\beta} N(t)^{\eta}-C(t) \leq K(t)^{\alpha} R(t)^{\beta} N(t)^{\eta}
$$

where $\eta=(1-\alpha-\beta)$. So, we get:

$$
\frac{\dot{K}(t)}{N(t)} \leq k(t)^{\alpha} r(t)^{\beta} \leq k(t)^{\alpha} R(t)^{\beta} \text { for all } t \geq 0
$$

And, since:

$$
\frac{\dot{K}(t)}{N(t)}=\dot{k}(t)+g(t) k(t) \text { for all } t \geq 0
$$

we obtain:

$$
\dot{k}(t) \leq k(t)^{\alpha} R(t)^{\beta}-g(t) k(t) \leq k(t)^{\alpha} R(t)^{\beta}
$$

This leads to:

$$
\dot{k}(t) / k(t)^{\alpha} \leq R(t)^{\beta} \text { for all } t \geq 0
$$

so that we obtain, after defining $z(t) \equiv k(t)^{1-\alpha} /(1-\alpha)$,

$$
\dot{z}(t) \leq R(t)^{\beta} \text { for all } t \geq 0
$$

Integrating (54) from 0 to $T>0$, we obtain:

$$
\begin{aligned}
z(T)-z(0) & \leq \int_{0}^{T} R(t)^{\beta} d t=\int_{0}^{T}\left[R(t)^{\beta}\right]\left[1^{1-\beta}\right] d t \\
& \leq\left[\int_{0}^{T} R(t) d t\right]^{\beta}\left[\int_{0}^{T} d t\right]^{1-\beta} \\
& \leq S^{\beta} T^{1-\beta}
\end{aligned}
$$

the second line in (55) following from Holder's inequality. Thus, there is a constant $A_{1}>0$, such that:

$$
k(T)^{1-\alpha} /(1-\alpha)=z(T) \leq A_{1} T^{1-\beta}
$$

and defining $(1-\alpha) A_{1}$ by $A_{2}$, we get:

$$
k(T)^{1-\alpha} \leq A_{2} T^{1-\beta}
$$

Finally, defining $A \equiv\left(A_{2}\right)^{1 /(1-\alpha)}$, we obtain:

$$
k(T) \leq A T^{(1-\beta) /(1-\alpha)} \text { for all } T>0
$$

Clearly, (56) yields the desired bound in (52). 


\subsection{A Restriction on the Technological Parameters}

Proposition 5 Let $(K(t), R(t), C(t))$ from $(K, S)$ in $\mathbb{R}_{+}^{2}$ be an arbitrary interior competitive equitable path. Then, the following technological restriction must hold:

$$
\alpha>\beta
$$

Proof. Let $(K(t), R(t), C(t))$ from $(K, S)$ in $\mathbb{R}_{+}^{2}$ be an arbitrary interior equitable path. We claim first the following (weaker) technological restriction must hold:

$$
\alpha \geq \beta
$$

To see this, note that we have for all $t \geq 0$,

$$
\begin{aligned}
\dot{k}(t) & \leq k(t)^{\alpha} r(t)^{\beta}-g(t) k(t)-c \\
& \leq k(t)^{\alpha} R(t)^{\beta}-c
\end{aligned}
$$

so that, using (56) yields:

$$
\begin{aligned}
\dot{k}(t) & \leq A^{\alpha} t^{\alpha[(1-\beta) /(1-\alpha)]} R(t)^{\beta}-c \\
& =A^{\alpha}\left[t^{\alpha /(1-\alpha)}\right]^{1-\beta} R(t)^{\beta}-c
\end{aligned}
$$

Then, integrating (58) from 0 to $T>0$ yields:

$$
\begin{aligned}
k(T)-k(0) & \leq A^{\alpha}\left[\int_{0}^{T}\left[t^{\alpha /(1-\alpha)}\right] d t\right]^{1-\beta}\left[\int_{0}^{T} R(t) d t\right]^{\beta}-c T \\
& \leq A^{\alpha}(1-\alpha)^{1-\beta} S^{\beta} T^{(1-\beta) /(1-\alpha)}-c T
\end{aligned}
$$

Thus, to keep $k(T) \geq 0$ for all $t \geq 0$, we must have (57) holding.

Now, we claim that the (stronger) technological restriction:

$$
\alpha>\beta
$$

must hold. To see this, note that (57) clearly holds. So, if (59) does not hold, we must have $\alpha=\beta$. Since $(K(t), R(t), C(t))$ is an interior, competitive and equitable path, (48) must hold. Thus, we have for $t \geq 0$,

$$
k(t)^{\alpha} r(t)^{\alpha}=k(t)^{\alpha} r(t)^{\beta} \geq c
$$

Now, by using (56) and $\alpha=\beta$, we obtain:

$$
R(t) \geq r(t) \geq \frac{c^{1 / \alpha}}{k(t)} \geq \frac{c^{1 / \alpha}}{A t}
$$

But, (60) violates the resource constraint (3)(b). Thus, (59) must hold. 


\subsection{A Quasi-Arithmetic Bound on Population Growth}

Theorem 2 Let $(K(t), R(t), C(t))$ from $(K, S)$ in $\mathbb{R}_{+}^{2}$ be an arbitrary interior competitive equitable path, with associated shadow prices $(p(t), w(t))$, given by $(6)$, and with constant per-capita consumption $(C(t) / N(t))=c>0$ for $t \geq 0$. Then, there exist constants $a, b>0$, such that:

$$
N(t) \leq a+b t^{\mu} \text { for all } t \geq 0
$$

where:

$$
\mu=\frac{(\alpha-\beta)(1-\beta)}{(1-\alpha) \beta}
$$

Proof. Since the path $(K(t), R(t), C(t))$ must satisfy the capital value transversality condition (by Proposition 1), we can find $T>0$, such that for all $t \geq T$,

$$
\beta(p(t) K(t))=\frac{K(t) R(t)}{K(t)^{\alpha} R(t)^{\beta} N(t)^{\eta}} \leq 1
$$

Thus, for $t \geq T$, we get:

$$
R(t) \leq \frac{N(t)^{\eta /(1-\beta)}}{K(t)^{(1-\alpha) /(1-\beta)}}
$$

Since (48) must hold (by Lemma 1), we have for $t \geq 0$ :

$$
K(t)^{\alpha} R(t)^{\beta} N(t)^{\eta} \geq c N(t)
$$

so that for $t \geq T$, using (61),

$$
\begin{aligned}
c N(t)^{1-\eta} & \leq K(t)^{\alpha} R(t)^{\beta} \\
& \leq K(t)^{\alpha-[(1-\alpha) /(1-\beta)] \beta} N(t)^{\beta \eta /(1-\beta)}
\end{aligned}
$$

This can be simplified to obtain for $t \geq T$,

$$
c N(t)^{\alpha /(1-\beta)}=c N(t)^{(1-\eta)-[\beta \eta /(1-\beta)]} \leq K(t)^{\alpha-[(1-\alpha) /(1-\beta)] \beta}=K(t)^{(\alpha-\beta) /(1-\beta)}
$$

Now, using (52) in (62), we obtain for $t \geq T$,

$$
c N(t)^{\alpha /(1-\beta)} \leq A^{(\alpha-\beta) /(1-\beta)} t^{(\alpha-\beta) /(1-\alpha)} N(t)^{(\alpha-\beta) /(1-\beta)}
$$

This yields for $t \geq T$ :

$$
c N(t)^{\beta) /(1-\beta)} \leq A^{(\alpha-\beta) /(1-\beta)} t^{(\alpha-\beta) /(1-\alpha)}
$$

which can be simplified to:

$$
N(t) \leq b t^{\mu} \text { for all } t \geq T
$$

where:

$$
b=\left[\frac{A^{(\alpha-\beta) / \alpha}}{c^{(1-\beta) / \alpha}}\right] \text { and } \mu=\frac{(\alpha-\beta)(1-\beta)}{(1-\alpha) \beta}
$$

Finally, denoting $N(T)$ by $a$, we have:

$$
N(t) \leq a+b t^{\mu} \text { for all } t \geq 0
$$




\section{Bibliography}

[1] Asheim, G.B., W. Buchholz and C. Withagen (2003), "The Hartwick Rule: Myths and Facts", Environmental and Resource Economics 25, 129-150.

[2] Asheim, G.B., W. Buchholz, J. M. Hartwick, T. Mitra and C. Withagen (2006) "Constant Savings Rates and Quasi-Arithmetic Population Growth under Exhaustible Resource Constraints", Journal of Environmental Economics and Management, forthcoming.

[3] Buchholz, W. (1982), "On the Possibility of Economic Growth with Exhaustible Resources" in Economic Theory of Natural Resources, (W.Eichhorn et. al. edited), PhysicaVerlag, Vienna, 295-299.

[4] Buchholz, W., S.Dasgupta and T.Mitra (2005), "Intertemporal Equity and Hartwick's Rule in an Exhaustible Resource Model", Scandinavian Journal of Economics, 107(3), 547-561.

[5] Cass, D. and T. Mitra (1991), "Indefinitely Sustained Consumption Despite Exhaustible Natural Resources", Economic Theory, 1, 119-146.

[6] Cunningham, F. (1967), "Taking Limits under the Integral Sign", Mathematics Magazine, 40 (1967), 179-186.

[7] Dasgupta, P.S. and G.M. Heal (1979), Economic Theory and Exhaustible Resources, Cambridge University Press, Cambridge.

[8] Dasgupta, S. and T. Mitra (1983), "Intergenerational equity and efficient allocation of exhaustible resources", International Economic Review, 24, 133-153.

[9] Dixit, A., P. Hammond and M. Hoel (1980) "On Hartwick's rule for regular maximin paths of capital accumulation and resource depletion", Review of Economic Studies, 47, $551-556$.

[10] Hartwick, J.M. (1977), "Intergenerational equity and investing of rents from exhaustible resources", American Economic Review, 66, 972-974.

[11] Kemp, M.C., N.V. Long and K. Shimomura (1984), "The Problem of Survival: A Closed Economy", in Essays in the Economics of Exhaustible Resources, (M.C. Kemp and N.V. Long, edited), North-Holland, Amsterdam, 13-26.

[12] Mitra, T. (1978a), "On Maintainable Consumption Levels and Exhaustible Resources", State University of New York at Stony Brook, Working Paper 198. 
[13] Mitra, T. (1978b), "Efficient growth with exhaustible resources in a neoclassical model", Journal of Economic Theory, 17, 114-129.

[14] Mitra, T. (1983), "Limits on Population Growth Under Exhaustible Resource Constraints," International Economic Review, 24, 155-168.

[15] Mitra,T. (2002), "Intertemporal equity and efficient allocation of resources", Journal of Economic Theory, 107, 356-376.

[16] Shimomura, K. (1983), "On the Survival Problem by Solow" (in Japanese), Kokumin Keizai Zasshi, 46, 51-65, Kobe University.

[17] Solow, R.M. (1974), "Intergenerational equity and exhaustible resources", Review of Economic Studies, 41, 29-45.

[18] Solow, R.M. (1986), "On the intergenerational allocation of natural resources", Scandinavian Journal of Economics, 88, 141-149.

[19] S. Takekuma (1982), "A Support Price Theorem for the Continuous Time Model of Capital Accumulation," Econometrica, 50, 427-442.

[20] Withagen, C. and G.M. Asheim (1998), "Characterizing sustainability: the converse of Hartwick's rule," Journal of Economic Dynamics and Control, 23, 159-165. 